\title{
A trajetória e a evolução da editoria científica em administração no Brasil: a proposta democrática do Cadernos EBAPE.BR
}

\author{
ISABElLa Francisca Freitas Gouveia de VASCONCELOS ${ }^{1}$ \\ HÉlio ARTHur REIS IRIgaray ${ }^{1}$ \\ FABIANA BRAGA LEAL ${ }^{1}$
}

${ }^{1}$ Fundação Getulio Vargas / Escola Brasileira de Administração Pública e de EMPresas, Rio de Janeiro - RJ, BRASIL

\begin{abstract}
Resumo
Este artigo apresenta brevemente o histórico e a evolução do periódico Cadernos EBAPE.BR, atualmente classificado como A2 no sistema brasileiro de avaliação de periódicos da Coordenação de Aperfeiçoamento de Pessoal de Nível Superior (Qualis/Capes). Destaca-se a preocupação da equipe editorial de seguir sua tradição ao publicar pesquisas bem fundamentadas e realizadas segundo critérios metodológicos rigorosos, considerando os valores democráticos ao permitir o debate acadêmico entre as várias correntes teóricas e escolas de pensamento da Administração de Empresas e tratando, especificamente, de temas como inclusão social, justiça social, inovação sustentável, desenvolvimento sustentável, teoria crítica e as possíveis interfaces desses temas com estratégia empresarial, gestão de pessoas e teoria das organizações.
\end{abstract}

Palavras-chave: Teoria crítica. Conhecimento. Administração de Empresas. Periódico científico.

History and evolution of scientific publishing in administration in Brazil: the democratic proposal of Cadernos EBAPE.BR

\begin{abstract}
This article presents a brief history and the evolution of the journal Cadernos EBAPE.BR, which is currently classified as A2 in the Brazilian evaluation system Qualis/Capes. The journal's editorial board is committed to the tradition of publishing well-grounded research with robust methodological criteria, observing democratic values that encourage academic debate between various theoretical currents and schools of scientific thought in the field of business administrations. The journal's publications emphasize themes such as social inclusion, social justice, sustainable innovation, sustainable development, critical theory, and the interfaces of these themes with business strategy, people management, and organization theory.
\end{abstract}

Keywords: Critical theory. Knowledge. Business Administration. Scientific journal.

Trayectoria y evolución de la publicación científica en el área de administración en Brasil: La propuesta democrática de Cadernos EBAPE.BR

\section{Resumen}

En este artículo presenta la reseña histórica y la evolución del periódico Cadernos EBAPE.BR, actualmente clasificado como A2 en el sistema brasileño de evaluación de periódicos de la Coordinación de Perfeccionamiento de Personal de Nivel Superior (Qualis/Capes). Se destaca la preocupación del equipo editorial de seguir su tradición, al publicar investigaciones bien fundamentadas y realizadas según rigurosos criterios metodológicos, considerando los valores democráticos al permitir el debate académico entre las diversas corrientes teóricas y escuelas de pensamiento de la Administración de Empresas y tratando, específicamente, temas como inclusión social, justicia social, innovación sostenible, desarrollo sostenible, teoría crítica, y las posibles interfaces de estos temas con estrategia empresarial, gestión de personas y teoría de las organizaciones.

Palabras clave: Teoría crítica. Conocimiento. Administración de empresas. Periódico científico. 


\section{INTRODUÇÃO}

Dois anos após a fundação da EBAPE em 1952, Benedicto Silva estabelecia a revista científica Cadernos de Administração Pública, que perdurou de 1954 até 1971, com incentivo do convênio entre FGV e a Fundação Ford. O objetivo era publicar artigos acadêmicos de qualidade para se criar pesquisa sobre conceitos e temas relevantes em Administração no Brasil. Em 1980 foi retomada a publicação do Cadernos de Administração Pública, com foco, então, em Administração de Empresas, em suas diversas áreas. Em 2003, com o Professor Dr. Marcelo Milano Falcão Vieira (período de atuação 2003 a 2009), o periódico passou à linha editorial atual como Cadernos EBAPE.BR, privilegiando o debate democrático de ideias, ensaios teóricos e pesquisas qualitativas.

O Prof. Marcelo Milano Falcão Vieira consolidou a revista e a diretriz era baseada na análise blind review - com publicações de alto nível acadêmico, sendo reconhecida pela comunidade acadêmica da área de Administração no Brasil e no exterior. $O$ corpo editorial da revista por ele proposto teve representação internacional com professores de várias escolas e continentes. A revista gozava de reconhecimento, contando com o apoio da equipe que atuava: Vania Mattos da Silva e Leonardo Vasconcelos Cavalier Darbilly.

Após o professor Marcelo Milano, seguiu como editora a Profa. Dra. Ana Lucia Malheiros Guedes (janeiro de 2010 a dezembro de 2012). Entre os muitos desafios que venceu com sucesso, tem-se a transição para o sistema on-line da Scielo Scientific Electronic Library. Neste período, além de manter o alto nível da revista e o reconhecimento da comunidade, a gestão da revista mostrou muita resiliência e determinação, uma vez que estava reduzida à editora-chefe e à assistente editorial Fabiana Braga Leal, ainda assim atingindo todos os objetivos propostos. Na gestão de Ana Lucia Guedes foram publicados números temáticos como aqueles dedicados ao LAEMOS (Latin American and European Meeting on Organization Studies), Interfaces Público-Privado no Contexto Luso-Brasileiro, Rio+20 dentre outros temas.

Na gestão do Prof. Dr. Fernando Guilherme Tenório (janeiro de 2013 a junho de 2016), a revista manteve a linha democrática de debate acadêmico entre diversas correntes da Administração, porém valorizando a Teoria Crítica, da qual Tenório é grande conhecedor. Foram também publicados números temáticos sobre Filosofia e Administração, Teoria Crítica e Habermas, Gestão Social e Sustentabilidade e Sustentabilidade e edição histórica tendo em vista o trabalho de Alberto Guerreiro Ramos.

No atual mandato, atuando como editores a Profa. Isabella Francisca Freitas Gouveia de Vasconcelos e o Prof. Hélio Arthur Reis Irigaray, dá-se a continuidade do trabalho empreendido por Tenório, com compartilhamento de muitos interesses acadêmicos. O Caderno EBAPE.BR, apoiado pela FGV, garante um debate acadêmico democrático, o interesse na Teoria Crítica, Habermas e Filosofia e outros temas ligados a gestão social, justiça social e inclusão social, sustentabilidade, inovação sustentável, valorizando pesquisas consistentes realizadas em todo o território brasileiro, representativas de realidades sociais do Norte, Centro-Oeste, Nordeste, Sudeste e Sul do País. Tem destaque também pela publicação de pesquisas de autores internacionais, visando garantir o intercâmbio de ideias. O corpo editorial é atualizado regularmente com o convite aos professores europeus e norte-americanos e mantendo a extensa representatividade na América Latina e no Brasil, bem como representantes de escolas asiáticas e também da Austrália.

Nesta gestão tem sido mantida a classificação Qualis CAPES (A2) obtida na gestão de Fernando G. Tenório, e objetivando maior visibilidade internacional as edições desde janeiro de 2018 são publicadas no formato bilíngue, almejando globalmente a comunidade acadêmica e a conquista de fator de impacto. Estamos prospectando a classificação da revista na lista da Fondation Nationale pour l'Enseignement de la Gestion des Entreprises (FNEGE), ligada ao Ministério da Educação da França e a migração para o sistema internacional ScholarOne Manuscripts.

Sua abrangência nacional/internacional e seu foco na interdisciplinaridade, com classificação Qualis Capes A2 na principal área de avaliação (Administração Pública e de Empresas, Ciências Contábeis e Turismo) e Qualis Capes B1 nas demais áreas do extrato (Artes, Ciências Agrárias I, Ciências Ambientais, Educação, Interdisciplinar, Linguística e Literatura, Planejamento Urbano e Regional - Demografia, Psicologia e Serviço Social, entre outras), são alguns dos aspectos que ratificam a singularidade do periódico Cadernos EBAPE.BR ao longo de seus 15 anos de existência, tendo em vista que:

a) ele vai ao encontro das perspectivas interdisciplinares e críticas nas quais sua linha editorial se baseia;

b) elabora chamadas de trabalhos com editores convidados nacionais e internacionais para a difusão e publicação de números temáticos abordando assuntos específicos junto à comunidade acadêmica; 
c) conta com um Conselho Editorial composto por membros nacionais e internacionais, responsáveis pela estrita revisão por pares (double blind review) implementada; e

d) proporciona acesso on-line e aberto para leitura/upload dos artigos tanto no portal do periódico como em suas bases de indexação.

O Cadernos EBAPE.BR tem apresentado significativa evolução desde seu surgimento até o ciclo corrente. Em 2003, as submissões totalizaram 48 artigos, passando para 241 em 2010 logo após a indexação no sistema da Scientific Electronic Library Online (SciELO) e atingindo 328 submissões em 2014; de janeiro a setembro de 2018 processou-se 248 artigos submetidos ao periódico. Também aumentou tanto a quantidade de submissões em inglês e espanhol como o número de artigos originais publicados nesses idiomas. O propósito atual é diminuir o tempo médio entre a submissão e o aceite do manuscrito e a publicação do artigo, proporcionando aos leitores estudos cada vez mais atualizados.

Quanto ao impacto dos artigos publicados, os dados bibliométricos são representados crescentemente pelo índice h5, que em 2013 foi 8, com mediana h5 = 9; em 2016, o índice h5 foi 12, com mediana h5 = 16; neste ano, o índice h5 tem sido 17, com mediana h5 $=22$. O fator de impacto no período de 2 anos, mensurado pelo indexador SciELO, foi 0.1949 para 2013; 0.2022 para 2014; 0.3077 para 2015; 0.1383 para 2016; e 0.2381 para 2017. O índice de citações recebidas e concedidas tem sido igualmente crescente.

\section{PUBLICAÇÕES CIENTÍFICAS E PRODUÇÃO DO CONHECIMENTO}

Os princípios fundamentais da ética segundo Habermas pressupõem a existência de uma comunicação entre diferentes sujeitos ao termo de uma discussão bem conduzida. Não há ética sem comunicação; e, especificamente, sem comunicação autêntica. A Teoria da Ação Comunicativa reúne, assim, Ética e Comunicação intersubjetivas dentro de um princípio de tolerância, democracia e ação política. Para que a ação comunicativa ocorra, além de seguir as regras de legitimidade explicitadas por Habermas, que devem ser aceitas pelo grupo social que interage, é fundamental que os membros do grupo que se comunica o faça com base em um princípio de igualdade de direitos. Como diz Apel (1987, p. 94), “É esta exigência de um reconhecimento recíproco das pessoas como sujeitos de uma argumentação lógica e não apenas o uso lógico correto da capacidade de compreensão do indivíduo que justifica que se fale de uma "ética da lógica".

Habermas distingue, assim, os três grandes critérios de uma verdadeira comunicação:

- ou o mundo em questão é objetivo, aquele do estado das coisas existentes, e pode gerar um saber partilhado que exige uma obrigação de justificação no que diz respeito à verdade dos fatos;

- ou o mundo é social (conjunto de relações interpessoais legitimamente estabelecidas no seio do grupo social) e o critério de validade é o da justiça (do que é justo);

- $\quad$ ou o mundo é subjetivo pessoal (conjunto de proposições relativas à experiência vivida), e o critério de validade é o de sinceridade.

Nos três casos, o ato de comunicação faz referência às normas comuns ao locutor e ao auditor, sem as quais não há como eles existirem de forma coerente e legítima.

A pesquisa bem realizada, baseada em uma metodologia consistente, que visa produzir conhecimento autêntico por meio de uma busca concreta da verdade dos fatos segundo a metodologia utilizada, o debate científico e acadêmico democrático entre várias correntes acadêmicas, com a garantia do double blind review e feedback dos pares para aperfeiçoamento dos artigos, o intercâmbio com diversas correntes de pensamento e pesquisa em congressos, reunindo pesquisadores de diversos países e culturas, são práticas essenciais para se preservarem valores fundamentais ao ser humano no seu processo de construção de conhecimento, inovação e na construção de uma sociedade mais justa.

Os periódicos científicos, ao publicar de forma transparente e democrática pesquisa autêntica e consistente, garantindo o debate científico, têm um papel fundamental no avanço do conhecimento de uma comunidade científica e de uma dada sociedade. 
Como dizem Berger e Luckmann,

O mundo da vida quotidiana não é considerado somente como um dado da realidade objetiva pelos membros de uma sociedade, tendo em vista a sua busca de sentido em suas vidas. Este mundo (o da vida quotidiana) também se origina em seus pensamentos e em suas ações e é mantido como realidade através deles. O pesquisador deve tentar esclarecer a origem do conhecimento na vida quotidiana, ou seja, as objetivações dos processos subjetivos e das significações que constroem o mundo do senso comum intersubjetivo (BERGER e LUCKMANN, 1989, p. 35).

Essa visão tem sua base em um conceito antropológico da realidade social (Administração pertence à área de Ciências Sociais Aplicadas), e, como mostra Linda Smircich em seus textos “Organizations as Shared Meanings (1983a)" e "Studying Organizations as Cultures (1983b)", busca regatar a visão específica de cada sujeito e de cada grupo organizacional, considerando que há várias racionalidades possíveis face a uma realidade, o que reforça o conceito da importância do debate democrático entre várias correntes de pensamento e pesquisa.

Nesse contexto, além de garantir a publicação democrática e a colaboração com o debate de ideias para a produção de conhecimento e o avanço dos conceitos em uma dada área, a importância dos periódicos científicos na área de Administração está em amparar/assistir o aprimoramento da pesquisa de autores de todo o país, através, por exemplo, das recomendações por avaliadores - professores e pesquisadores, pares com igual titulação e conhecimento durante a análise do artigo na fase blind review, propondo os aprimoramentos à pesquisa aos pesquisadores/autores.

A revista, divulgando a pesquisa científica, segue a mesma linha aplicada pelo Egos (European Group of Organizational Studies), o qual define da seguinte maneira seus objetivos:

A EGOS é uma associação acadêmica que promove o avanço teórico e/ou empírico do conhecimento sobre organizações, como se estruturam e os contextos nos quais operam. Como um coletivo, um de seus principais objetivos é manter e proporcionar uma voz para as abordagens críticas e analíticas de seus membros no estudo das organizações em todo o mundo.

A EGOS tem sua identidade e raízes intelectuais nas ciências sociais. A associação encoraja uma abordagem analítica e teórica em relação às organizações, acolhendo a diversidade em todas as suas formas, incluindo uma abordagem plural da compreensão das organizações na perspectiva das ciências sociais (como a sociologia, a história social, ciência política, psicologia e a antropologia), bem como das ciências humanas (como a filosofia, a análise de discurso, a crítica literária e a retórica).

A associação oferece um fórum para identificar e discutir questões-chave na teoria e na prática organizacional. (Tradução nossa) (EGOS).

Egos foi fundado em 1973 e influenciou o Prof. Marcelo Milano Falcão, instituidor deste periódico, e os intelectuais brasileiros que seguem a corrente do pensamento crítico e analítico da ANPAD (Associação Nacional de Pesquisa em Administração), assim como os editores que atuaram na revista: Ana Lucia Malheiros Guedes e Fernando Guilherme Tenório e os presentes editores Isabella F. F. G. Vasconcelos e Hélio Arthur Reis Irigaray.

Nessa mesma linha, identificamo-nos com o Critical Management Study (CMS) Division of Academy of Management, que expressa seus objetivos da seguinte forma:

A Critical Management Studies Division (Divisão de estudos críticos de gestão) é um fórum dentro da Academy of Management (Academia de gestão) onde se pode expressar opiniões críticas sobre práticas de gestão antiéticas e que exploram a ordem social, trabalhando para que essas práticas sejam mudadas. (Tradução nossa) (CMS).

Ou seja, busca criticar as práticas de abuso contra os direitos humanos e as minorias e denunciar práticas não éticas propondo políticas de transformação dessa realidade para uma realidade social mais justa.

Nesse sentido, a divisão 'Organizations and the Environment', da Academy of Management, define seus objetivos como: 
Domínio específico: pesquisa, teorias e práticas sobre as relações das organizações e o ambiente natural. Os principais tópicos incluem: sustentabilidade ecológica, filosofias e estratégias ambientais, desempenho ecológico, empreendedorismo ambiental, indústrias de produtos e serviços ambientais, controle e prevenção da poluição, minimização de resíduos, ecologia industrial, gestão ambiental de qualidade total, auditoria ambiental e sistemas de informação, gestão de recursos humanos para sustentabilidade, gestão de crises ecológicas, gestão de recursos e sistemas naturais, proteção e restauração, interações de gestão de sistemas, interações de stakeholders ambientais, políticas ambientais, atitudes ambientais e tomada de decisão, e dimensões internacionais/comparativas destes tópicos. (Tradução nossa).

São temas que nós, na revista, privilegiamos, porque, como bem observado pela Organização das Nações Unidas (ONU) e reforçado no Acordo de Paris sobre o meio ambiente, as práticas que o protegem tendem a gerar trabalho e atividades que levam a uma maior justiça social, inclusão social e respeito aos direitos humanos. O Cadernos EBAPE.BR vem publicando, nessa linha, muitos artigos sobre sustentabilidade e responsabilidade social e inclusão.

A ADERSE (Association pour le developpement de l'Etude de la Responsabilité Sociale D'Entreprises - www.aderse.org.br) é um congresso que também trata destes temas correlatos.

Em relação ao futuro, há uma busca por maior justiça e sustentabilidade na economia mundial.

A Conferência da IFSAM (International Federation of Schorlaly Associations of Management), que visa ser uma ONU das diversas associações de management no mundo, tratou desse tema (www.ifsam.org), organizando, em 2010, um congresso intitulado "Justice and Sustainability in a Global Economy". Também exclusivamente voltado a esse assunto, a Academy of Management realizou o congresso "Green Management Matters".

Mais tarde, o desenvolvimento dessa área de pesquisa e a realização da Cúpula de Paris mostraram que se busca caminhar em pesquisa e ação social nesse sentido.

O envolvimento da iniciativa privada também nessa direção e o desenvolvimento de pesquisa aplicada nessa linha parecem ser fundamentais neste contexto para fazer prosperar as políticas de sustentabilidade.

Diante disso, os periódicos científicos têm o seu importante papel ao divulgar teoria e também pesquisa aplicada de qualidade e o Cadernos EBAPE.BR vem cumprindo o seu papel nesse sentido.

Periódicos como Organizational Studies, Academy of Management Review, vêm se dedicando há anos em fazer evoluir a pesquisa científica no Brasil e integrá-la com os principais centros de pesquisa do mundo. Pretendemos continuar nesta direção! 


\section{REFERÊNCIAS}

APEL, K. O. L'éthique à I'âge de la science. Lille: PUF, 1987.

BERGER, P.; LUCKMANN, T. A Construção Social da Realidade. Petrópolis: Vozes, 1989.

CMS. Critical Management Studies. Disponível em: $<\mathrm{http}: / / \mathrm{cms}$.aom. org/about-the-division/>. Acesso em: 24 out. 2018.

EGOS. European Group for Organizational Studies. Disponível em: <https://www.egosnet.org/egos/about_egos>. Acesso em: 24 out. 2018.
SMIRCICH, L. Organizations as Shared Meanings. In: PONDY, L.; FROST, P.J.; MORGAN, G.; DANDRIDGE, T.C. (Ed.). Organizational Symbolism. Greenwich, Connecticut: JAI Press, 1983a. p. 56-65.

SMIRCICH, L. Studing Organizations as Cultures. In: MORGAN, G. (Ed.). Beyond Method for Social Research. Bervely Hills: CA, Sage, 1983b. 424p.

Isabella Francisca Freitas Gouveia de Vasconcelos

Doctorat es Sciences de Gestion, HEC-Ecole des Hautes Etudes Commerciales, França (2000); Doutorado em Administração de Recursos Humanos, FGV EAESP (1997); Pós-Doutorado em Rutgers the New Jersey State University; Professora Adjunta da FGV EBAPE; Pesquisadora no CNAM Conservatoire National D’Arts et Metiers, França. E-mail: isabella.vasconcelos@fgv.br

Hélio Arthur Reis Irigaray

Doutor e Mestre em Administração de Empresas pela FGV EAESP e PUC-Rio, respectivamente; Bacharel em Economia pela University of Northern Iowa, EUA; Professor adjunto da FGV EBAPE e do programa CIM - Corporate International Masters, da Georgetown University, Washington, EUA; Líder do tema Diversidade e Relações de Trabalho, na linha de Gestão de Trabalho (ANPAD). E-mail: helio.irigaray@fgv.br

Fabiana Braga Leal

Mestranda em Administração - Mestrado Executivo em Gestão Empresarial na Fundação Getulio Vargas / Escola Brasileira de Administração Pública e de Empresas. E-mail: fabiana.leal@fgv.br 